\title{
Comparing Infrared Thermography and ESPI for NDE of Aircraft Composites
}

\author{
Dirk Findeis, Jasson Gryzagoridis, Chilufya Lombe \\ Department of Mechanical Engineering, \\ University of Cape Town \\ Private Bag, Rondebosch, 7700, South Africa \\ Tel: +27 216503670 \\ Dirk.findeis@uct.ac.za
}

\begin{abstract}
Infrared Thermography is gaining acceptance by industry as a suitable non destructive testing and evaluation (NDT/E) technique. Likewise Electronic Speckle Pattern Interferometry (ESPI) has also been shown to be suited for similar purposes, particularly for the inspection of composite materials.

This paper presents the theory of the two inspection methods. Selected composite samples containing defects are subjected to identical inspection procedures using both techniques. The results are presented and compared with each other. From the results it is clear that both techniques are suited for the detection of the defects in the composite sample. The results also indicate that ESPI is a more sensitive inspection technique than IRT. Comparisons are drawn and the benefits of each technique discussed.
\end{abstract}

\section{Introduction}

Structures and components made from composite materials are being developed and manufactured in many diverse industries due to their high strength to weight ratio, resistance to degradation, structural rigidity and ease of shaping. In the commercial aircraft arena Airbus and Boeing are taking full advantage of the benefits of composite materials with the new Boeing Dreamliner aircraft reportedly having a 50\% composite content. Composites however are susceptible to damage during the manufacturing phase and also whilst in service. Very often this damage is not readily visible to the naked eye and is present below the surface of the object. If left undetected the defect could slowly increase in size and weaken the structure which ultimately could lead to catastrophic failure. It is thus important to develop and specify suitable inspection techniques which can be applied without altering the integrity of the inspected component or structure, or changing its properties. Two such techniques which can be used for the detection of flaws in composites and are potentially useful in the aerospace industry ${ }^{1,4,5,6}$ are Infrared Thermography (IRT) and Electronic Speckle Pattern Interferometry (ESPI). The range of defects detectable include, but are not limited to: delamination, debonding and fracture in composite laminate parts, delamination, debonding or object damage due to foreign objects as well as liquid contamination and corrosion in composites. 


\section{Theory}

IRT is used for the non destructive localization of thermally resistive zones within an object, as often is the case when a defect is present. The technique makes use of the fact that all materials above zero Kelvin will radiate electromagnetic energy, some of which is in the infra red wavelength range. The magnitude of this fraction of energy is directly proportional to the object surface emissivity and temperature. Because this wavelength range is not visible to the naked eye, a thermal imager is used to measure the magnitude of the emission and convert this to a visual image. In the image each radiation energy level is characterized by either a colour, or a grey scale level ${ }^{1}$. In other words, images presented in the video feed from the camera are a record of the temperature variations of the surface and can range either from white for warm regions to black for cooler areas on a black and white image or in colours ranging from reds to blues using a pseudo colour system. As radiation does not require a medium for transfer, there is no need for a thermal imager to be in contact with the surface being recorded.

As IRT records the radiation emission characteristic of an object, the technique is generally divided into 2 categories. Passive thermography is the application of IRT which relies on the component or structure to generate it's own thermal energy due to some mechanical, chemical or electrical process. Active thermography on the other hand is the application of IRT in the presence of some form of external stimulation to generate the thermal gradient within the inspection object. In most cases this is the preferred method of inspection of inert components.

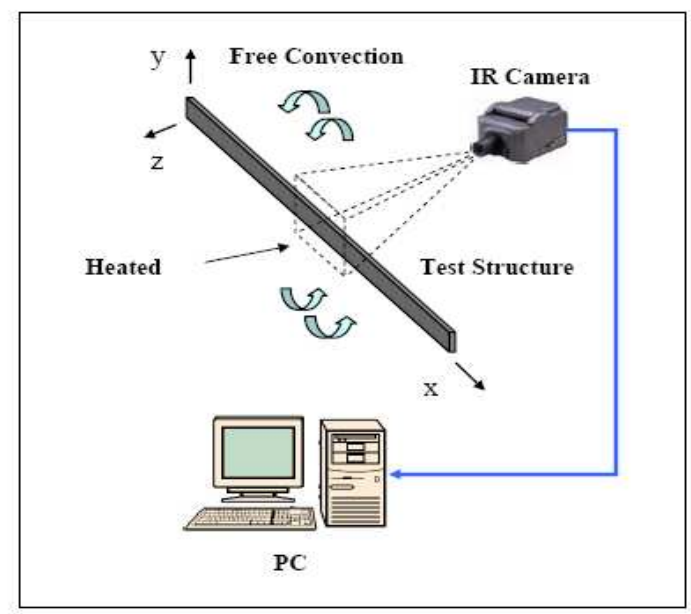

Figure 1. IRT application schematic

For active thermography, uniform heating of a homogenous material would result in a uniform temperature distribution. If an object has an internal defect or material anomaly, the thermal resistance to heat flow is altered in the region of the defect ${ }^{2}$. When the object to be inspected is heated and the resulting transient heat transfer rate monitored, the localised variation in the thermal resistance due to the defect produces a temperature differential between the defect and the rest of the specimen, which directly affects the magnitude of the thermal radiation off the surface of the object. The heating of the material can be done with quartz lamps or hot air from a dryer and applied either 
continuously or in a pulsed manner using flash lamps, either in transmission or reflection mode.

Images produced by the thermal imager can be interrogated to detect localized areas of differing temperatures, which can be related to the presence of either surface or subsurface material or structural variations. In order to capture and process these images, infrared cameras are often connected to and driven by a computer. Figure $1^{3}$, shows a typical setup of an IRT system.

ESPI on the other hand is based on the recording of speckle images, created when two light waves interfere with one another. In order for this to occur, the light waves have to be monochromatic, which is why single mode lasers, both continuous and pulsed, are used for this NDE inspection technique. ESPI produces fringe patterns that depict an object's surface displacement in response to an applied stress, which can be produced via thermal, pressure, vacuum or mechanical means. The magnitude of the applied stress is very small and produces an object surface displacement of only a couple of micrometers. In order to elaborate on how this is achieved, the following schematic in figure 2 has been included.

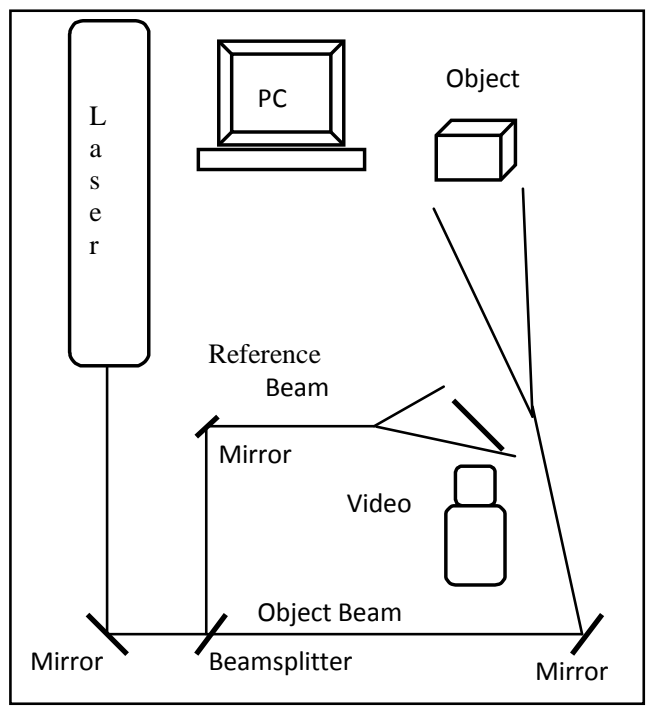

Figure 2. ESPI setup schematic

Figure 2 outlines a typical out-of-plane displacement ESPI set-up. A laser beam is split into two with the aid of a beamsplitter. The first beam, called the object beam and consisting of approximately $90 \%$ of the available laser energy, is expanded via a beam expander before being used to illuminate the object to be inspected. A CCD camera is then focused onto the object to capture the monochromatic reflection off the object. The second beam, called the reference beam is expanded and directed onto a beamsplitter, placed in front of the camera, in order to direct the reference beam onto the camera's CCD chip. If the beam path lengths of the reference and object beams are within the coherent length of the laser, the two light waves will interfere to produce a speckle pattern. By connecting the camera to a PC equipped with a suitable framegrabber, the video signal can be digitized, stored and processed. 
When an object is stressed, either mechanically or thermally, the object surface deflects. This causes the laser beam path length used to illuminate the object to change. The associated change in phase of the laser light also causes the speckle interference pattern to change. By capturing the speckle interference pattern of the unstressed object and comparing it with the speckle interference pattern of the stressed object, it is possible to locate regions of correlation and decorrelation between the two images. This produces a familiar zebra-like fringe pattern. For ESPI this can be represented mathematically by equation 1 below $^{7}$.

$d=\frac{n \lambda}{[\cos \alpha+\cos \beta]}$

where: $\quad d=$ out of plane displacement of the object due to the applied stress,

$\alpha=$ angle between the camera viewing angle and normal to the object,

$\beta=$ angle between the object beam direction and normal to the object,

$\lambda=$ wavelength of the laser beam,

$\mathrm{n}=$ no of fringes counted.

As the wavelength of the laser beam is fixed, equation 1 reveals that the magnitude of the surface displacement is directly related to the number of correlation fringes.

\section{Results}

In order to use the two inspection techniques a section of an Oryx helicopter rotor blade was provided. The blade construction consists of two outer fiberglass skins which are wrapped around a shaped Nomex honeycomb core. Additionally a stainless steel skin is bonded over the leading edge. The upper and lower skins are bonded together at the trailing edge of the rotor blade.

Three circular $42 \mathrm{~mm}$ defects of varying depth were introduced into the sample section by cutting away the skin and a certain amount of the Nomex honeycomb to varying levels of depth, as listed in the table below.

Table 1. Details of artificially created flaws

\begin{tabular}{|c|c|c|c|}
\hline Defect & Depth of Defect & $\begin{array}{l}\text { Overall Honeycomb } \\
\text { Thickness }\end{array}$ & $\begin{array}{l}\text { Thickness of Remaining } \\
\text { Honeycomb }\end{array}$ \\
\hline Defect 1 & $20 \mathrm{~mm}$ & $27 \mathrm{~mm}$ & $7 \mathrm{~mm}$ \\
\hline Defect 2 & $30 \mathrm{~mm}$ & $49 \mathrm{~mm}$ & $19 \mathrm{~mm}$ \\
\hline Defect 3 & $25 \mathrm{~mm}$ & $51 \mathrm{~mm}$ & $26 \mathrm{~mm}$ \\
\hline
\end{tabular}

The front and rear side of the prepared sample section can be seen in the accompanying figure 3 below. Here the position of the defects can be clearly identified from the one side, but not the other. 


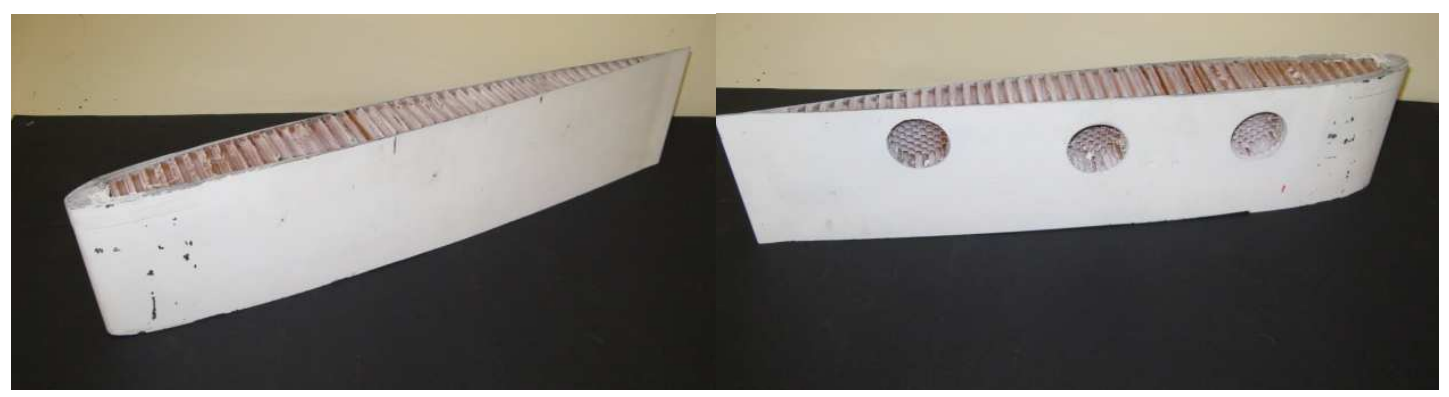

Figure 3. Oryx Rotor blade section with three artificial defects: front and back view

It was decided to apply active thermal heating for the NDE inspection methods. A standard hairdryer clamped in a retort stand and connected to a timer was used. This ensured repeatability of the thermal load for both inspection techniques. In order to test the sensitivity of both methods the heating duration of the object was kept short, namely 0.5 seconds, 1 second and 1.5 seconds. The alternative would have been to apply a longer heating period and let the object relax back to room temperature, whilst monitoring the image output to determine the threshold levels. It was felt that this would be a subjective approach and hence the former was selected. The laboratory setup for the ESPI Setup can be seen in figure 4 below. For the IRT inspection the location of the sample and heater remained the same but the laser was switched off and the ESPI camera was replaced with an IRISYS 1000 Series thermal imaging camera.

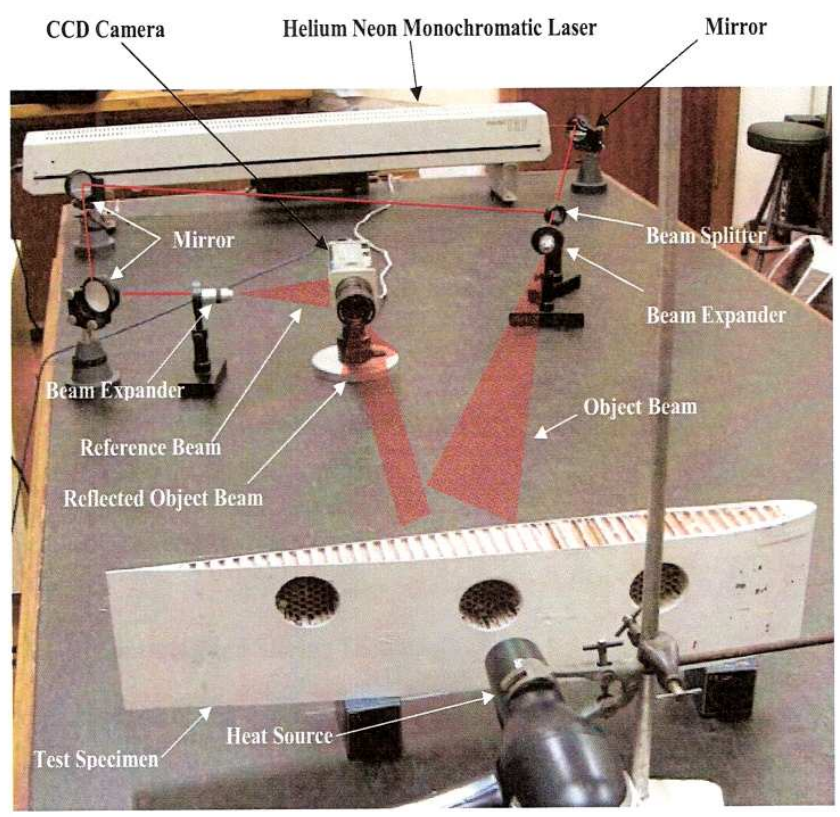

Figure 4. Laboratory ESPI and sample setup

The results of the inspections are presented below. For each defect and heating period, the results of the ESPI and IRT have been grouped together for comparison purposes. The ESPI results are listed on the left and the IRT colour image of the same defect on the right of the combined figures. For defects 2 and 3, the intermediate heating result has been left out, but is recorded in table 2 later. 


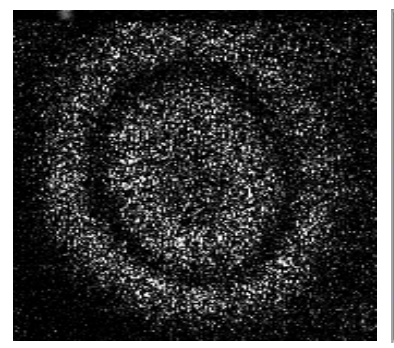

Figure 5. Defect 1, 0.5s heating
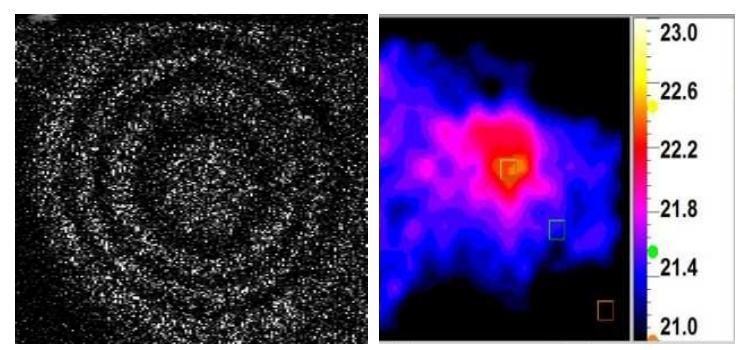

Figure 6. Defect 1, 1.0s heating
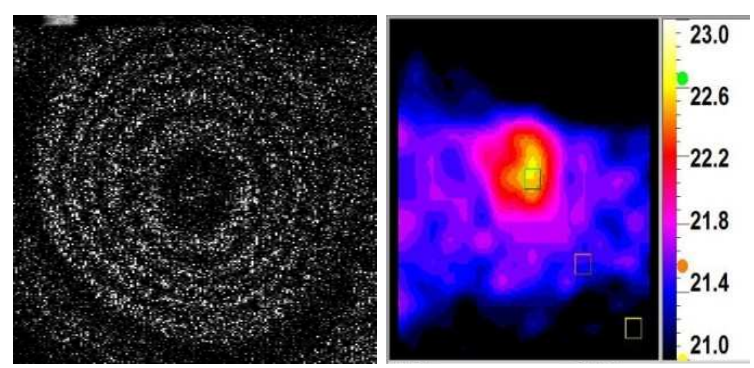

Figure 7. Defect $1,1.5 \mathrm{~s}$ heating
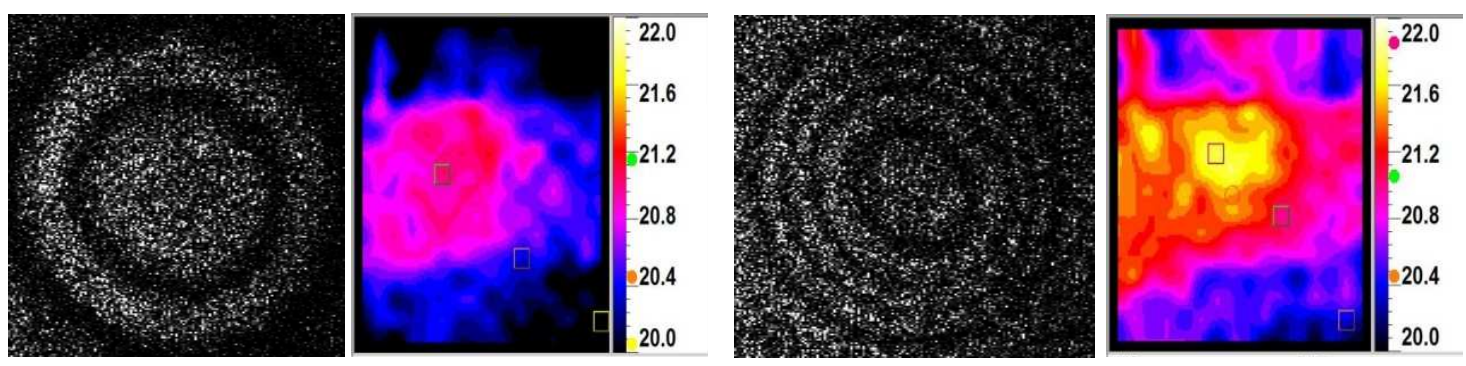

Figure 8. Defect 2, 0.5s heating

Figure 9. Defect 2, 1.5s heating
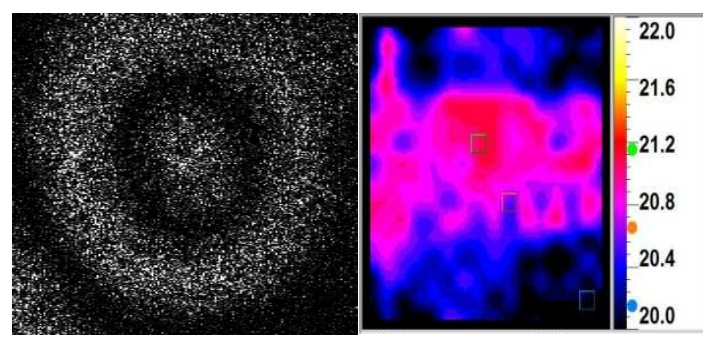

Figure 10. Defect 3, 1.0s heating

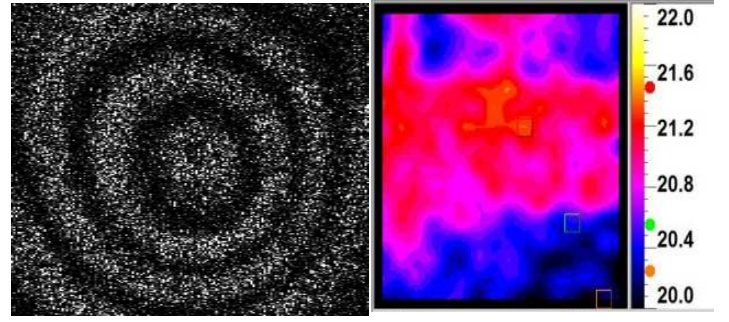

Figure 11. Defect 3, 2.0s heating

Figure 5 to figure 11 above depict the results obtained. In all of them the location of the defect can be detected in either the circular ESPI fringe pattern or via the IRT colour image depicting the temperature distribution across the image surface. When comparing the results of figure 5,6 and 7, it is apparent that there is a direct relationship between the duration of heat applied and the resultant number of fringes in the ESPI result, and the final maximum temperature in the IRT image. Figure 5 has the least and figure 7 the most. 
There appears to be a relationship between the depth of the defect and the number of fringes produced when comparing figure 5 with figure 8 and figure 10 . The result of figure 10 is for a 1 second heating period, as 0.5 seconds was found to be too little to produce a usable IRT result. This is more readily apparent when comparing figures 7, 9 and 11, which is the result for the maximum heating times for each defect. The fringes recorded were 5, 3 and 2 fringes respectively, and the associated temperatures $22.7^{\circ} \mathrm{C}$, $22^{\circ} \mathrm{C}$ and $21.5^{\circ} \mathrm{C}$. Defect 1 had the least amount of honeycomb left and defect 3 the most.

A closer look at the results of figure 10 indicate that ESPI was able to clearly detect the presence of the defect with a 1 second heating period but the IRT results are not as clear and the shape of the defect cannot be clearly defined. This indicates that ESPI is more sensitive than the IRT camera used for this inspection procedure.

Table 2. Table of the combined ESPI and IRT fringe and temperature results

\begin{tabular}{|c|c|c|c|c|}
\hline Defect & Heat Supplied (s) & $\begin{array}{c}\text { Remaining Thickness } \\
\text { of Honeycomb }\end{array}$ & $\begin{array}{c}\text { Number } \\
\text { of Fringes }\end{array}$ & $\begin{array}{c}\text { Hotspot } \\
\text { Temperature }\left({ }^{\circ} \mathrm{C}\right)\end{array}$ \\
\hline Defect 1 & 0.5 & 7 & 1 & 22.1 \\
\hline Defect 1 & 1 & 7 & 3 & 22.5 \\
\hline Defect 1 & 1.5 & 7 & 5 & 22.7 \\
\hline Defect 2 & 0.5 & 19 & 1 & 21.2 \\
\hline Defect 2 & 1 & 19 & 2 & 22 \\
\hline Defect 2 & 1.5 & 19 & 3 & 21.9 \\
\hline Defect 3 & 1.0 & 26 & 1 & 21.2 \\
\hline Defect 3 & 1.5 & 26 & 1 & 21.3 \\
\hline Defect 3 & 2.0 & 26 & 2 & 21.5 \\
\hline
\end{tabular}

Table 2 above is very handy for comparing the results obtained from the two inspection techniques and summarizes and supports the comments made above. The only result which does not follow the trends discussed above is the IRT result for defect 2 with 1 second heating applied. At $22^{\circ} \mathrm{C}$ peak temperature this reading is greater than the reading for the same defect with 1.5 seconds heating. It is possible that some form of experimental error crept into that particular inspection sequence.

\section{Conclusions}

From the above results it is clear that both techniques are able to detect defects exposed to low levels of active thermal loading and are thus both suitable for NDE of composite materials.

ESPI is more sensitive than IRT at it was able to clearly locate all defects present at all thermal loading conditions. The IRT results were not conclusive for all inspection scenarios, in particular defect no 3 with the thickest honeycomb section remaining. A temperature difference was noted but no distinctive hotspot formed. 
There is a clear relationship between the increase in thermal loading magnitude and the resultant increase in surface temperature for IRT and number of fringes formed using ESPI. Likewise for identical thermal loading conditions, there is a clear relationship between the reduction in number of fringes and surface temperature obtained and the increase in honeycomb material remaining below the defect.

\section{Recommendations}

Even though it is indicated that ESPI is the more sensitive of the 2 NDE techniques investigated, it does not mean that it is a better technique than IRT. The technique chosen is directly related to the application. For field work, the ease of use of the IRT camera makes it better suited than the ESPI configuration. On the other hand if the object to be inspected is not suited for thermal loading inspections, IRT is not a method to be considered. For laboratory conditions ESPI would be the preferred inspection method due to the greater sensitivity of the technique and low levels of object stressing required.

\section{References}

[1] Meola, C. and Carlomagno, G. M., 2004, "Recent Advances in the use of Infrared Thermography," Measurement Science and Technology. 15, pp 27-58.

[2] Khan, A. U. Md., 1999, "Non-destructive Testing Applications in Commercial Aircraft Maintenance," Proc. 7th European Conference on Non-destructive Testing, Copenhagen, 26-29 June, Vol 4(6).

[3] Cecchini, A., 2005, "Damage Detection and Identification in Sandwich Composites using Neural Networks," MSc. Thesis, University of Puerto Rico.

[4] Wong, B.S., Tu, C.G., Bai,W., Tan, P.H., Low, B.S., and Tan,K.S.,(n.d) "Thermographic Evaluation of Defects in Composite Materials," http://www.ntu.edu.sg/mae/Research/programmes/Sensors/NDT/thermographyb yBSWong.pdf.

[5] Gryzagoridis, J., and. Findeis, D., 2005, "Simultaneous Shearographic and Thermographic NDT of Aerospace Materials," www.ndt.uct.ac.za.

[6] Thomas, R.L., and Favro L.D, 1998, "Thermal Wave Imaging of Fluid Intrusion in Composites," http://www.eng.wayne.edu/legacy/imr/NASAFAADoD98.pdf.

[7] R Jones, C Wykes, Holographic and Speckle Interferometry, $2^{\text {nd }}$ Ed. Cambridge University Press, 1989 